\title{
Correction to: MicroRNA-9 regulates survival of chondroblasts and cartilage integrity by targeting protogenin
}

Jinsoo Song ${ }^{1+}$, Dongkyun Kim ${ }^{1 \dagger}$, Churl-Hong Chun ${ }^{2}$ and Eun-Jung Jin ${ }^{1 *}$

Correction to: Cell Commun Signal (2013) 11:66

http://www.biosignaling.com/content/11/1/66

Following publication of the original article [1], the authors reported that Figs. 3 and 6 are incorrect.

There are errors in a Safranin O staining image for DMM/miR-9 in Fig. 6e and Alcian blue staining images for control and miR-9 in Fig. 3a. The correct figures are supplied below.

\section{Author details}

'Department of Biological Sciences, College of Natural Sciences, Wonkwang

University, Iksan, Chunbuk 570-749, South Korea. ${ }^{2}$ Departments of

Orthopedic Surgery, Wonkwang University School of Medicine, Iksan,

Chunbuk 570-749, South Korea.

Published online: 28 November 2019

\section{Reference}

1. Song J, et al. MicroRNA-9 regulates survival of chondroblasts and cartilage integrity by targeting protogenin. Cell Commun Signal. 2013;11:66 http:// www.biosignaling.com/content/11/1/66.

Full list of author information is available at the end of the article 

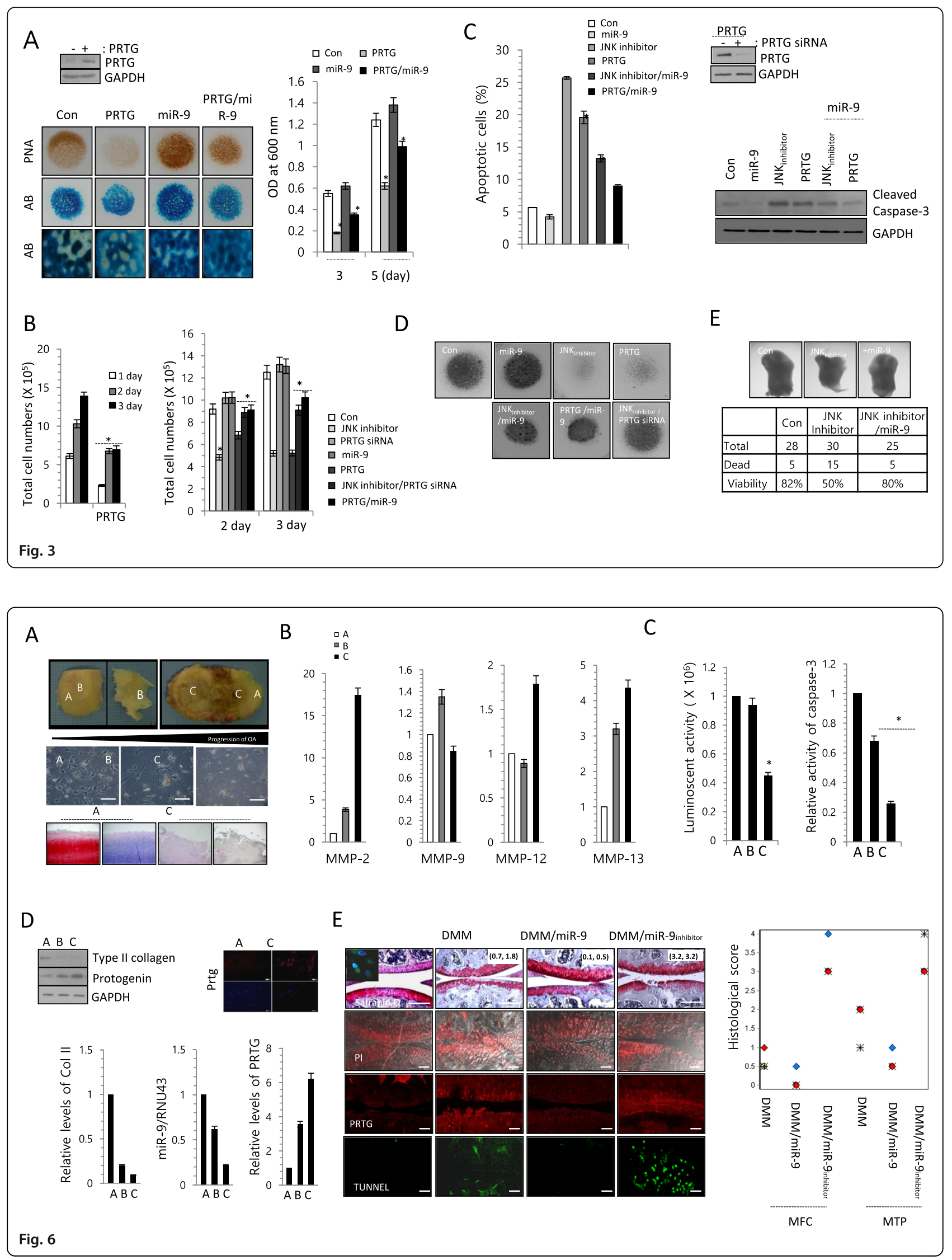